\title{
An Atomistic View of Electrodics at the Platinum
}

\section{Surface}

Sumit Bawari, * Tharangattu N. Narayanan, * and Jagannath Mondal*

Tata Institute of Fundamental Research-Hyderabad, Sy. No. 36/P Serilingampally Mandal, Ranga Reddy District, Hyderabad - 500046, India.

KEYWORDS: Platinum; Electrodics; Reactive Force Field; Molecular Dynamics; Platinum Leaching.

\section{ABSTRACT}

Platinum (Pt) is a benchmarked catalyst for several electrochemical processes, however an atomistic insight into its electrodics at the electrode-electrolyte interface is still lacking. In this study, we aim to capture the chemical changes of Pt surfaces brought on by an applied potential in an electrolyte of $\mathrm{pH} \sim 5$, which can address the catalytic efficacy and stability of different crystallographic orientations under varying applied bias. Through a combined experimental and reactive molecular dynamics simulation approach, we uncover the effect of charge build up on the surface of the Pt electrode, which can be directed towards capacitive and faradaic processes. By introducing a simulated applied potential, which is compared to experimental potential by equating charge density ( in the range $-0.2 \mathrm{mC} / \mathrm{cm}^{2}$ to $0.2 \mathrm{mC} / \mathrm{cm}^{2}$ ), we unravel the electrochemical processes on Pt (in slightly acidic $\mathrm{pH}$ ). At reductive potentials of $\sim 0.3-0.0 \mathrm{~V}$ vs RHE, we visualize 
phenomenon such as under potential hydrogen adsorption ( $\mathrm{H}_{\mathrm{UPD}}$ ) and hydrogen evolution/oxidation reaction. While oxidative potentials in the range $\sim 1.2-1.6 \mathrm{~V}$ vs RHE see platinum oxide (Pt-O) formation, and platinum leaching off the surface. The theoretical potential and plane dependence of these phenomenon (HUPD, Pt-O, etc.) are verified with experiments, and hence it brings a new platform for computationally viable electrode-electrolyte studies.

\section{Introduction}

Electrocatalysis is a promising alternative to traditional thermal catalysis, and has huge importance in energy-related as well as small molecule catalysis. ${ }^{1,2,3}$ Heterogeneous electrocatalytic processes such as hydrogen evolution reaction (HER) (counter reaction: oxygen evolution reaction (OER)) and hydrogen oxidation reaction (HOR) (counter reaction: oxygen reduction reaction $(\mathrm{ORR})$ ) are important for the generation of hydrogen and further fuel cell fueling up on protons, respectively. ${ }^{1}$ Electrochemical processes are also playing pivotal roles in the reduction of $\mathrm{N}_{2}$ and $\mathrm{CO}_{2}$ to produce value added products. ${ }^{2,3}$ Since all these reactions take place in electrolytes, the electrode-electrolyte interface plays an important role in determining the dynamics of heterogeneous electrocatalytic processes. ${ }^{4}$ Although numerous catalysts have been reported for such processes, an atomistic understanding on the electrode-electrolyte interfacial phenomenon is still lacking. ${ }^{4}$ This is particularly due to the complex nature of the multi-step processes, where different time and length scales are involved, making the computational analyses complicated. ${ }^{5}$ Understanding these interfacial processes are highly important to verify the stability and long term performance of the catalyst in electrolyte.

To understand the electrocatalysis process, various computational strategies have been proposed that go beyond an adsorption based free-energy calculations. ${ }^{6}$ It is known that a typical electrochemical process begins when an external potential greater than the equilibrium potential 
of the reaction is applied. In a heterogeneous catalytic process: the electroactive molecule gets adsorbed on the catalyst followed by its bonding to the surface. Further charge transfer steps catalyze the adsorbed reactant, and the product (s) is desorbed. Density functional theory (DFT) based gas phase free energy calculations are predominantly reported to determine the reaction intermediates, which can explain the bonding efficacy of the reactants to the surface. ${ }^{7}$ A mirror charge assisted DFT calculations have been reported to simulate the charging process through the application of an external potential. ${ }^{7}$ However, the overall system - including counter ions and solvent, becomes very computationally expensive for density functional theory (DFT) based calculations and hence DFT based studies cannot simulate the long timescale dynamics of the system.

In this stead, molecular dynamics (MD) simulations provide alternative simulation strategies where diffusion dynamics can be studied. ${ }^{5}$ In MD, classical force fields can be adapted to simulate an external potential and study capacitive layer formation. ${ }^{8}$ However, electrochemical reactions require the modeling of bonding interactions along with diffusion and an external potential, to get the complete microscopic picture of the electrodics during catalysis. In this study, we aim to simulate the bonding interactions along with ion dynamics and external potential in the platinum (Pt)-electrolyte system, where plentiful experimental data is available. ${ }^{9} \mathrm{Pt}$ is an ideal catalyst for the HER and OER processes, with the lowest required overpotential. ${ }^{9}$ Cheng et. al. have shown the applicability of the reactive force field (ReaxFF) force field in simulating a Pt/water interface. ${ }^{10}$ By applying the EChemDID method, developed by Onofrio et. al.,${ }^{11,12}$ where it can simulate an external potential by changing the electronegativity of certain atoms, here we simulate a $\mathrm{Pt} /$ electrolyte $(\mathrm{pH} \sim 5)$ system with an external potential. 
Even though Pt is one of the most explored electrochemical surfaces, the changes in chemical species, which can activate or hinder Pt's further activity towards electrocatalytic processes are not well understood yet. At reductive (cathodic) potentials, under potential deposited hydrogen (HUPD), which is a preceding step for hydrogen evolution, is known to follow a plane dependent behaviour. ${ }^{9}$ The nature of HUPD is studied here on different Pt surfaces (crystallographic orientations/planes), and their potential dependence is uncovered. At oxidative (anodic) potentials, a surface alpha- $\mathrm{PtO}_{2}$ formation has been shown using in situ Raman spectroscopy. ${ }^{13}$ While at higher anodic potentials, three-dimensional oxides of Pt can be formed, where they act as sites for the OER process at higher bias voltages. However, these oxides have been predicted to cause leaching of Pt atoms from the electrode,${ }^{14}$ and this leaching process also follows a plane dependent behaviour. The electrochemistry with the exposed planes of lower coordination, Pt-110 and Pt100, show much more leached content of Pt in the electrolyte than that of highly coordinated Pt111 surface, as studied by in situ inductively coupled-atomic emission spectroscopy (ICP-AES) experiments. ${ }^{15}$ Simulated platinum structures at high oxygen content show a leaching in the form of small clusters of platinum oxides $\left(\mathrm{Pt}_{6} \mathrm{O}_{8}{ }^{4-}\right)$, which may get detached during electro-catalysis. ${ }^{20}$ Even though oxide formation contributes to this process, leaching can also occur before the onset of platinum oxidation, which is not well studied yet. By simulating the Pt/electrolyte system we observe the HUPD and PtO intermediates, which are followed by HER and OER, respectively. At oxidative potentials, Pt leaching is also seen in this study, which correlates well with recent studies. An atomistic view and mechanisms of relevant processes are discussed in detail.

\section{Results and Discussion}


Platinum electrochemistry: The cyclic voltammetry (CV) response of a poly-crystalline platinum electrode in $\mathrm{pH} \sim 5(0.1 \mathrm{M}$ acetate buffer and scan rate $200 \mathrm{mV} / \mathrm{s})$ is shown in figure $1 \mathrm{~A}$ (details are given in the methods section). The $\mathrm{pH} \sim 5$ is chosen to correlate the experimental results with the simulated data.
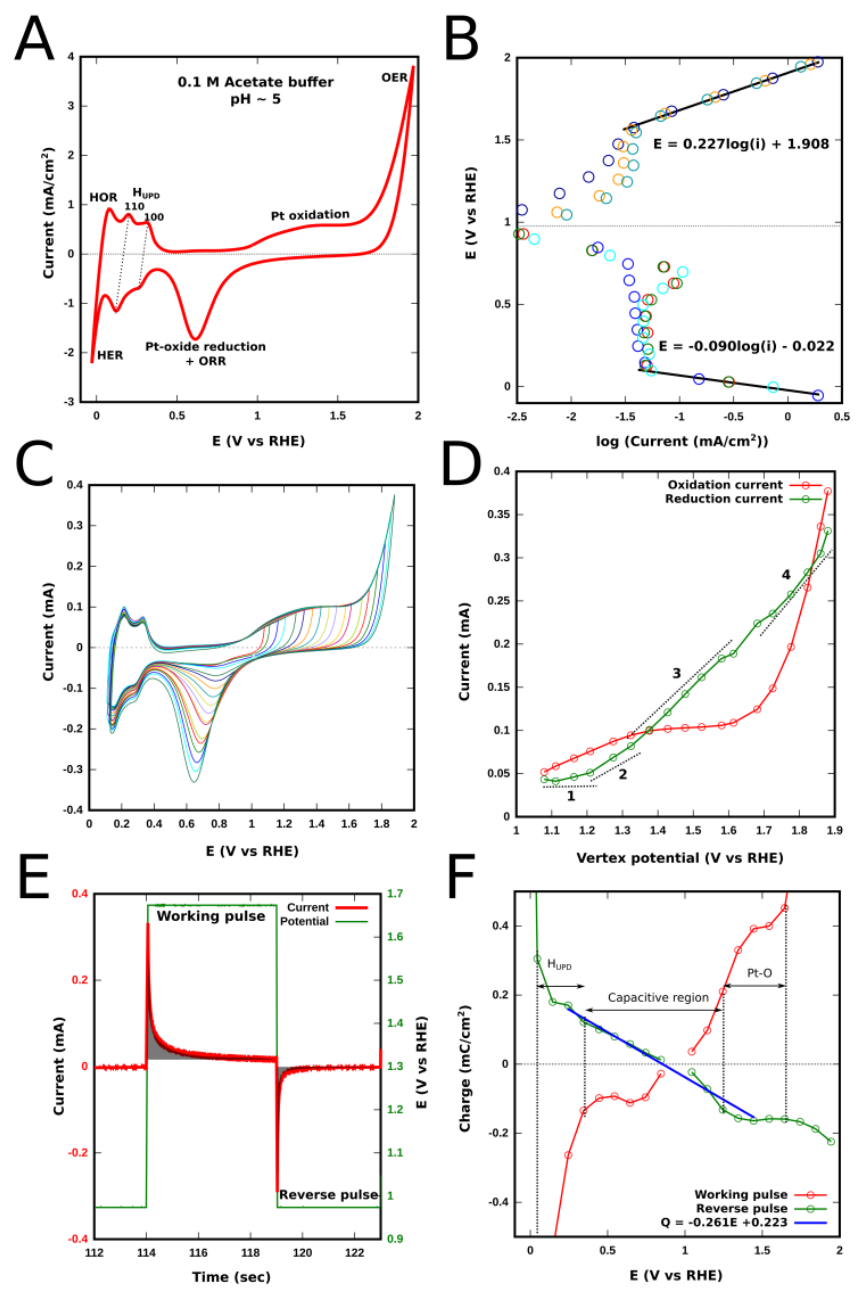

Figure 1: A) The Cyclic voltammogram (CV) of a polycrystalline platinum electrode with the details of the electrochemical processes mentioning. Scan rate is $200 \mathrm{mV} / \mathrm{s}$ and the electrolyte is $0.1 \mathrm{M}$ acetate buffer $(\mathrm{pH} \sim 5)$. B) Tafel plots with slopes for OER and HER are shown. C) CVs of platinum and oxidation reduction processes, scanned till certain vertex potentials (from 0.9 to 1.9 V). D) Current derived from figure 1C for oxidation current at specific vertex potentials, and the corresponding peak reduction response at $0.6-0.8 \mathrm{~V}$. E) Representative pulse voltammetry: I-t and 
$\mathrm{V}$-t profiles showcasing the working and reverse potential pulse, and a current profile with the shaded region implying charge integration. F) Charge calculated by integrating the current decay after a potential pulse (working pulse), and the reverse pulse to OCV (0.9 V vs RHE).

The CV (figure 1A) shows characteristic features of Pt at different potential windows. ${ }^{21} \mathrm{In}$ the oxidation region, we see a broad current peak $(\sim 1.2 \mathrm{~V})$ which appears when the surface platinum gets oxidized, after which the oxygen evolution reaction $(\mathrm{OER})$ starts $(\sim 1.6 \mathrm{~V})$. At negative potentials, a current peak that can be ascribed to $\mathrm{PtO}$ reduction $(\sim 0.7 \mathrm{~V})$ can be seen. At higher negative potentials, specific current peaks corresponding to $\mathrm{H}_{\mathrm{UPD}}$ can be seen for the crystal planes of Pt-110 ( 0.1 V) and Pt-100 ( 0.3 V). Beyond these potentials, the HER (and HOR) processes $(\sim 0 \mathrm{~V})$ start to contribute to the current. While Pt oxidation/reduction, HER/HOR and OER/ORR are faradaic processes, Pt also forms a directed capacitive interface with water preceding these faradaic reactions. The tafel plot can be extracted from the equilibrated current in a $\log (\mathrm{I}) v s$ applied bias (V) plot, shown in figure 1B. The tafel plot for both the oxidation and reduction regions can be divided into three regions: (i) formation of double layer capacitance with water, (ii) preferential Pt-H (H $\left.\mathrm{H}_{\mathrm{UPD}}\right)$ and Pt-O formation, and (iii) HER/OER onset. The oxidative and reductive voltage sweeps followed by the stabilization of the electrochemical system is found to shift the initial open circuit voltage (OCV, $0.9 \mathrm{~V})$ reversibly. An oxidised working electrode has a higher OCV, while a reduced electrode attains a lower OCV value. A change in OCV during oxidation suggests chemical changes which occur on the electrode with stable surface platinum oxide formation, that can be reduced in subsequent reduction steps. 
To quantify the potential at which $\mathrm{PtO}$ formation starts, we monitor the peak reduction current near the area where $\mathrm{PtO}$ reduction is expected, as the potential is swept till different vertex potentials in the oxidation region (figure 1C). The oxidation current at different potentials and the corresponding peak reduction current are plotted in figure 1D, which show four regimes of oxidation. Region 1 - from $\sim 1.05$ to $\sim 1.2 \mathrm{~V}$ : the oxidation current increases steadily while no significant increment is apparent in the PtO reduction peak. Region 1 can be ascribed to a charged bi-layer formation with water. Region 2 - from $\sim 1.2$ to $\sim 1.3 \mathrm{~V}$ : an onset in the PtO reduction current can be seen, while the oxidation current continues to increase linearly and reaches a plateau. Region 3 - from $\sim 1.3$ to $\sim 1.6 \mathrm{~V}$ : a constant oxidation current is maintained while PtO reduction current increases linearly. Region 4 - above $\sim 1.7$ V: OER takes over and thus the reduction current is a combination of PtO reduction and ORR. These results indicate that the Pt oxidation occurs in region 2 and 3 (above $\sim 1.2 \mathrm{~V}$ ), with different reaction kinetics.

The charge accumulated on the electrode can be determined by using pulse voltammetry and integrating the current response. ${ }^{16}$ Starting at the OCV $(\sim 0.9 \mathrm{~V})$, potential steps of $100 \mathrm{mV}$ are taken for both oxidation and reduction steps. A typical pulse cycle is shown in figure 1E, where the potential is changed from $0.95 \mathrm{~V}(\mathrm{OCV})$ to $1.65 \mathrm{~V}$. The initial pulse is called the 'working pulse', while the second pulse is called the 'reverse pulse'. In the working pulse ( 0.95 to $1.65 \mathrm{~V})$, charge is built up on the electrode for processes that can occur at $1.65 \mathrm{~V}$ (double layer capacitance, oxidation, OER, etc.). While in the reverse pulse (1.65 to $0.95 \mathrm{~V})$, the reverse processes are expected to occur to bring the electrode to its initial state. Since at OCV $(\sim 0.95 \mathrm{~V})$, PtO reduction and ORR - which are the reverse processes of Pt oxidation and OER, cannot occur, hence the reverse charge will only contribute to the reversal of the charged double layer. In this way, charge originating from faradaic and capacitive processes can be decoupled from each other through pulse 
voltammetry. The difference in the charge (shaded regions) is apparent in figure 1E, and the same procedure is applied to calculate the charge in both oxidative and reductive potentials (figure 1F). In purely capacitive regions near OCV, we could see that both working and reverse pulse charges are similar in magnitude. In the faradaic process regions, the working pulse charge increases drastically while the reverse pulse charge stagnates. As charge is expected to follow a linear dependence on external potential, ${ }^{16}$ we linearly fit the capacitive region to obtain a relation between capacitive charge and external potential as, $Q\left(\mathrm{mC} / \mathrm{cm}^{2}\right)=-0.261 * E(V)+0.223$. This relation is used to scale the simulated potential from the observed charge in the next section.

\section{Simulating the Electrodics at Pt:}

a) Charge Accumulation and Crystallographic Orientation Dependency: A system consisting of two platinum electrodes, in carbonic acid ( $\mathrm{pH} \sim 5)$, are used to model the electrochemical system studied here (figure 2A, details are provided in the methods section). With ReaxFF parameters for $\mathrm{Pt} / \mathrm{C} / \mathrm{H} / \mathrm{O}$, the EchemDID module is used to simulate an external potential of $\mathrm{V}_{\text {sim }}$ between the two platinum electrodes. ${ }^{17,11}$ Protruding conical electrodes are used to simulate a rugged surface which is closer to a real polycrystalline platinum surface. These polycrystalline surfaces contain local regions which can be ascribed to different Pt crystallographic orientations (planes), Pt-111, 100, and 110 , as shown in figure $2 \mathrm{~B}$ (ii). The simulated external potential, $\mathrm{V}_{\text {sim }}$, manifests in the form of charge accumulation on the surface of the catalyst surface. An external potential of 0 to $-8 \mathrm{~V}_{\text {sim }}$ (cathode) and 0 to $+8 \mathrm{~V}_{\text {sim }}$ (anode) is applied on the electrode, above which the interactions seen in simulations are deemed unrealistic. This may be due to the fact that we do not directly observe any faradaic reactions (HER/OER), and only the preceding step potentials can be simulated here. $\mathrm{V}_{\text {sim }}$ defines a change in the empirical electronegativity $\left(\chi_{0}\right)$, and it cannot be directly equate to 
the actual voltage. To be able to correlate with experimental potential, the acquired charge in simulations is used as a measure.

The simulated charge shows an almost linear response within the charge range between -0.2 and $0.2 \mathrm{mC} / \mathrm{cm}^{2}$ (figure $2 \mathrm{C}$ ), which can be compared to the range $-0.28 \mathrm{mC} / \mathrm{cm}^{2}$ and $0.20 \mathrm{mC} / \mathrm{cm}^{2}$ seen for the capacitive regions (figure 1D) in experiments. Since the magnitude of charge accumulation (in $\mathrm{mC} / \mathrm{cm}^{2}$ ) is comparable between experiments and simulations, we use the relation shown in figure $1 \mathrm{D}$ to scale the simulated potential $\mathrm{V}_{\text {sim }}$ as, scaledsimulationpotential $=3.83 *$ $Q\left(\mathrm{mC} / \mathrm{cm}^{2}\right)+0.85$ (table 1$)$. From this scaling relation between $\mathrm{V}_{\text {sim }}$ (which determines charge (Q)) and experimental potential, the $0 \mathrm{~V}_{\text {sim }}$ falls close to $0.82 \mathrm{~V}$ vs RHE, which is close to the experimental OCV value. The charge accumulation across the surface of the electrode at positive and negative potentials can be seen in figure $2 \mathrm{~B}$. In the subsequent sections, we look at the oxidation and reduction regions as modeled in these simulations, and try to match the onset of different reactions as seen in experiments.

Table 1: Scaling of simulation potential, using charge acquired from simulations and chargepotential relation in figure $1 \mathrm{~F}$.

\begin{tabular}{|r|r|r|r|r|}
\hline $\mathrm{V}_{\text {sim }}$ & $\begin{array}{r}\mathrm{Q} \\
\text { (anode) }\end{array}$ & $\begin{array}{r}\mathrm{Q} \\
\text { (cathode) } \\
\mathrm{mC} / \mathrm{cm}^{2}\end{array}$ & $\begin{array}{r}\text { Scaled simulation } \\
\text { potential, anode } \\
\mathrm{mC} / \mathrm{cm}^{2}\end{array}$ & $\begin{array}{r}\text { Scaled simulation } \\
\text { potential, cathode }\end{array}$ \\
\hline 0 & -0.01 & -0.01 & 0.83 & $\mathrm{~V} v$ s $\mathrm{RHE}$ \\
\hline 1 & 0.01 & -0.02 & 0.89 & 0.82 \\
\hline 2 & 0.02 & -0.04 & 0.94 & 0.77 \\
\hline 4 & 0.05 & -0.08 & 1.05 & 0.70 \\
\hline 5 & 0.08 & -0.10 & 1.14 & 0.54 \\
\hline 6 & 0.10 & -0.13 & 1.25 & 0.46 \\
\hline
\end{tabular}




\begin{tabular}{|r|r|r|r|r|}
\hline 7 & 0.14 & -0.19 & 1.40 & 0.14 \\
\hline 8 & 0.20 & -0.26 & 1.61 & -0.15 \\
\hline
\end{tabular}
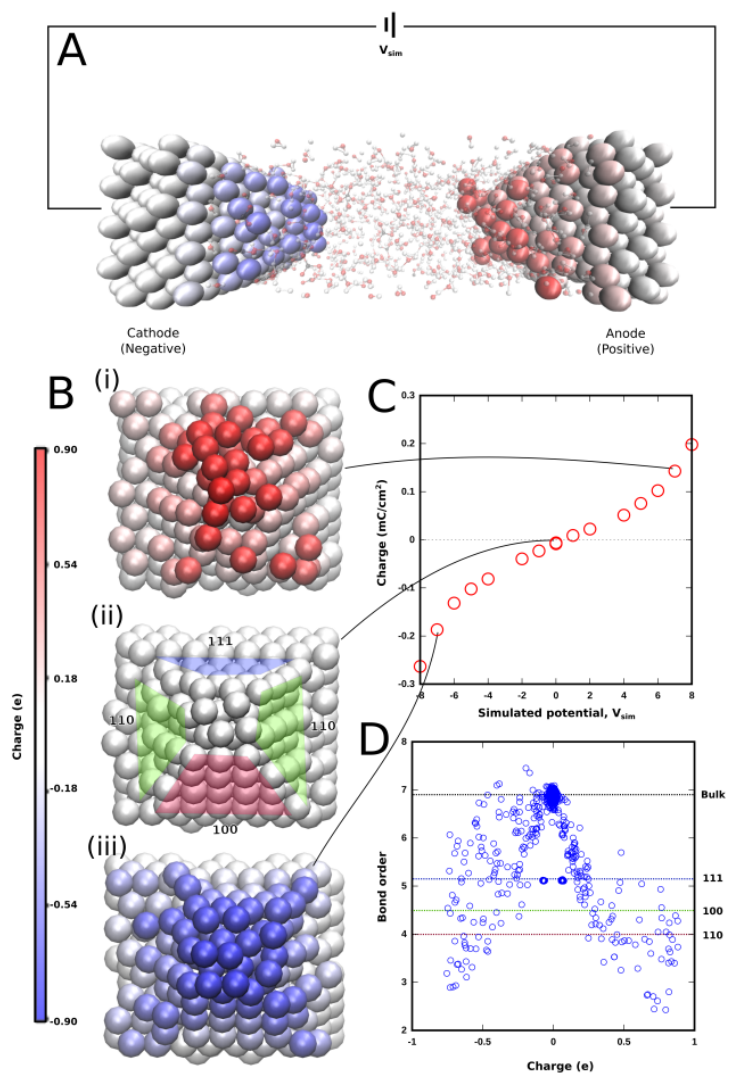

Figure 2: A) Schematic of the simulation setup with a platinum cathode and anode separated by an electrolyte, $\mathrm{V}_{\text {sim }}$ denotes the potential applied. B) Morphology and charge distribution of the conical polycrystalline platinum electrode at different potentials $\left(+7 \mathrm{~V}_{\mathrm{sim}}(\mathrm{i}), 0 \mathrm{~V}_{\mathrm{sim}}(\mathrm{ii}),-7\right.$ $\left.\mathrm{V}_{\text {sim(iii) }}\right)$. C) Total charge accumulated on each electrode at different simulated potentials. D) Dependence of charge on the bond order of an atom at a particular potential, $7 \mathrm{~V}_{\text {sim. }}$.

Another aspect that can be studied in simulations is the plane dependent behaviour of different interactions. Figure 2D shows a clear dependence of accumulated charges on the bond order of the platinum atom in question. Atoms buried inside the electrode, which form the bulk phase, have no 
charge on them, showing that only the surface gets charged. This charge is bond-order dependent, and increases with decreasing bond order. The accumulated charge follows the following order (activity in descending order with the one highest first) on the planes of polycrystalline platinum, Pt-110 $>$ Pt-100 $>$ Pt-111. The edges that form between these phases accumulate even more charge, as they have lower coordination. A notable difference in spread of charge is also seen between the positive (anode) and negative (cathode) electrodes, where negative charge is more delocalised than positive charge.

b) The Structure of Water Near the Electrodes: The overall charge (and current) in the presence of an external potential, induces the formation of capacitive interfaces and bonding interactions. The radial distribution functions (RDF) for Pt-H and Pt-O interactions are shown in figure 3. At $0 \mathrm{~V}$, a peak in the Pt-O interaction at $\sim 2.1 \AA$ shows the first solvation layer, with a second solvation layer at $\sim 3.1 \AA .{ }^{10}$ These two layers appear to form a buckled bilayer of water, at no external voltage, where a difference of $\sim 1 \AA$ is expected between the two water layers. ${ }^{18}$ The presence of a peak at $2.1 \AA$ for both Pt-O and Pt-H can be ascribed to, either O-H planes ( $1^{\text {st }}$ layer) parallel to the surface of platinum or directed $\mathrm{O}-\mathrm{H}$ planes ( $2^{\text {nd }}$ layer) to the platinum surface. On the anode, the Pt-O (at $2.1 \AA ̊$ ) peak intensity increases with increasing potential showing the buildup of a $\mathrm{Pt}-\mathrm{OH}_{2}$ capacitive layer. Two peaks in the Pt-H RDF, which can be ascribed to hydrogen atoms facing the platinum surface $(\sim 2.1 \AA)$ and hydrogen atoms facing away from the platinum surface $(\sim 2.5 \AA)$ show reverse behavior on the cathode and anode, and the relative occupancy of these can explain the first solvation layer's behaviour. A decrease in the Pt-H $2.1 \AA / 2.5 \AA$ ratio suggests the 
reorientation of water surface to compensate higher number of oxygen atoms. The positively charged $\mathrm{Pt}$ surface forms a dipole with $\mathrm{O}-\mathrm{H}_{2}$, to provide a capacitive response.
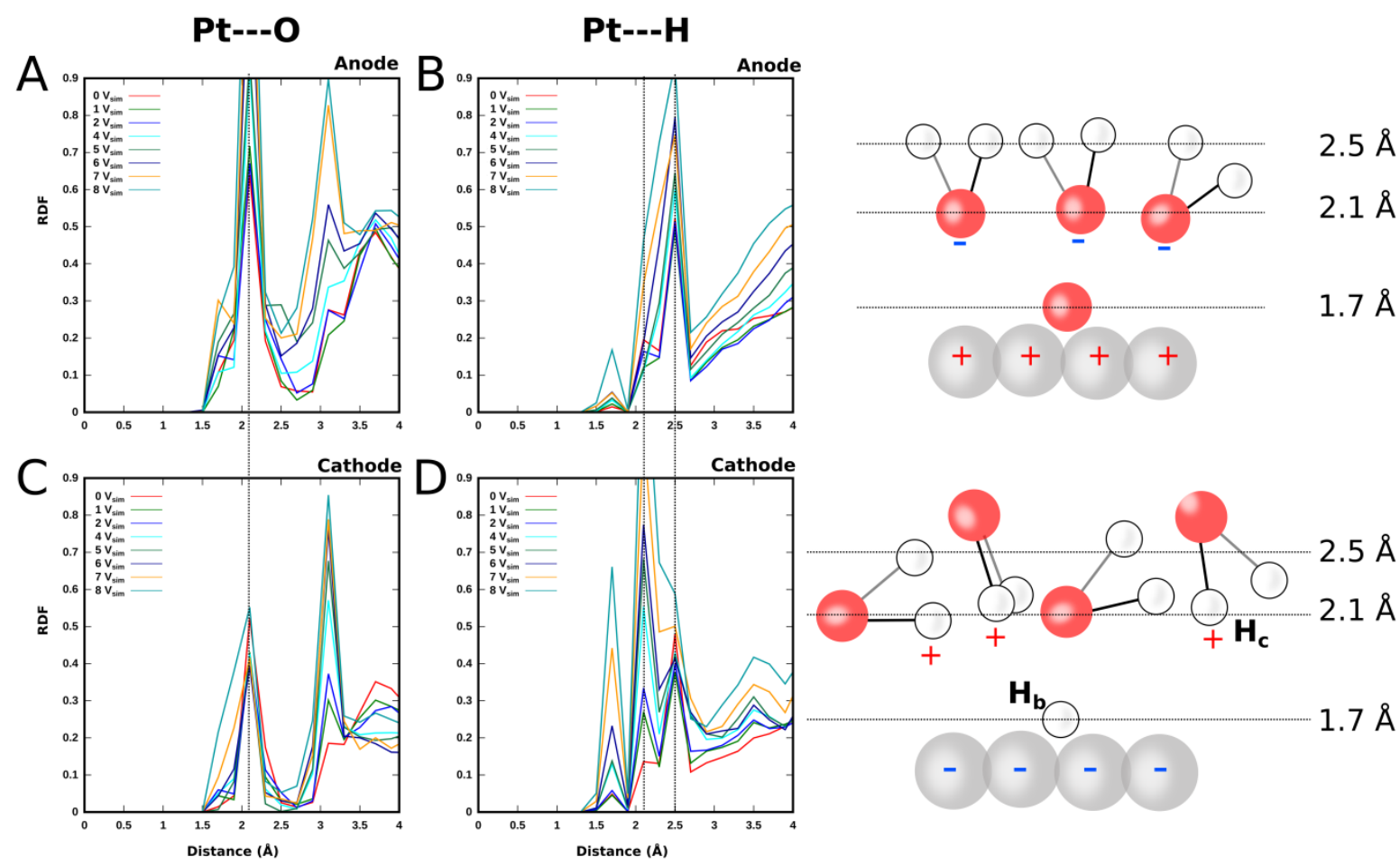

$1.7 \AA$

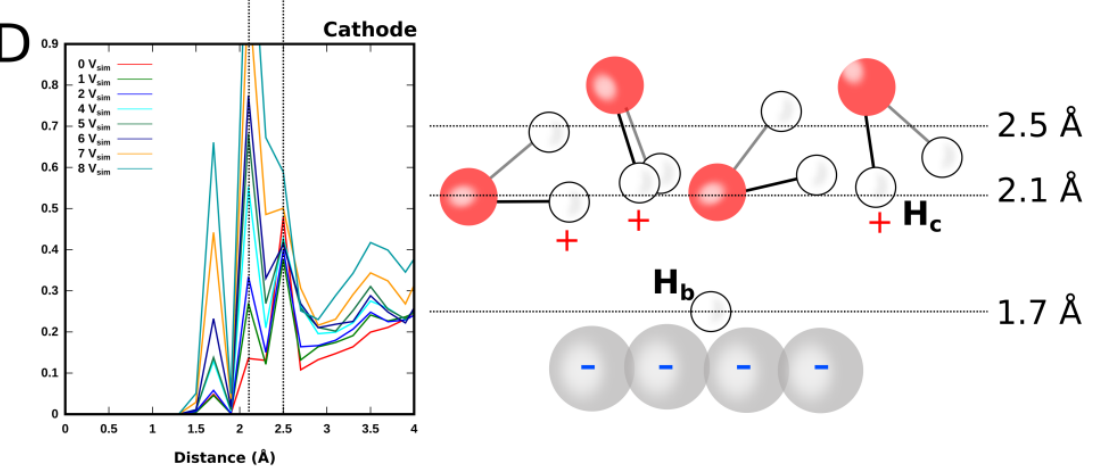

Figure 3: Radial distribution functions (left) between Pt and O (A,C), and between Pt and H (B,D) for the anode $(\mathrm{A}, \mathrm{B})$ and cathode $(\mathrm{C}, \mathrm{D})$ at different potentials $\left(\mathrm{V}_{\text {sim }}\right)$ with distances of 2.1 and 2.5 $\AA$ marked. Accompanying schematics (right) depicting water structure near the anode and cathode, with $\mathrm{H}_{\mathrm{c}}$ (capacitive hydrogen) and $\mathrm{H}_{\mathrm{b}}$ (bonded hydrogen) marked.

Contrary to the anode, the Pt-O peak at $2.1 \AA$ shows little change with increasing potential, while an increase in the $3.1 \AA$ peak is observed. Here the $2.1 \AA / 2.5 \AA$ ratio increases with potential, suggesting the formation of a greater number of directed Pt..H-OH bonds from the second water layer. This interface is closer to the flat ice structure expected for Pt-111/water interfaces. These 
directed Pt..H-OH bonds form a capacitive response on the electrode. At distances closer than $\sim 1.8$ $\AA$, bonding interactions take over.

c) Cathodic Reactions- UPD and HER: On the cathodic side, two types of hydrogen species can be seen (figure 3 schematic), bound Pt-H $\left(\mathrm{H}_{\mathrm{b}}\right)($ at $\sim 1.7 \AA)$ and $\mathrm{Pt}-\mathrm{H}^{+} \mathrm{OH}\left(\mathrm{H}_{\mathrm{c}}\right)$ forming a capacitive layer (at $\sim 2.1 \AA$ ). If we look at the increase in the $\mathrm{H}_{\mathrm{b}}$ and $\mathrm{H}_{\mathrm{c}}$ type species with scaled simulation potential, we can see an almost linear increase in both (figure $4 \mathrm{~A}$ ). The line at $\sim 0.4 \mathrm{~V}$ marks the capacitance to $\mathrm{H}_{\text {UPD }}$ transition as observed in the experimental data (figure $1 \mathrm{~A}$ ). The $\mathrm{H}_{\mathrm{c}}$ is seen to increase drastically from 0.85 to $0.4 \mathrm{~V}$, at which points it reaches a high coverage. The $\mathrm{H}_{\mathrm{b}}$, however, only starts building up after $\sim 0.4 \mathrm{~V}$, which dictates that bound hydrogen $\left(\mathrm{H}_{\mathrm{b}}\right)$ corresponds to the HUPD peak. The $\mathrm{H}_{\mathrm{b}}$ seen here corresponds to the bound hydrogen atoms that sit in adsorption sites shown in figure $4 \mathrm{C}$. At higher coverage, many of the $\mathrm{H}_{\mathrm{b}}$ atoms can also penetrate into the second layer of platinum, forming subsurface hydrogen species.

In the polycrystalline conical structure (figure 2B(ii)), the different sides of the cone consist of localised planes, Pt-111, 100, and 110. The Pt HUPD response has been well studied for the Pt planes 111, 100, and 110. Pt-100 and Pt-110 show specific peaks pertaining to the filling of UPD sites, while Pt-111 has no peak pertaining to HUPD, meaning a slow and constant filling of UPD sites. Decoupling the $\mathrm{H}_{\mathrm{b}}$ on different planes, $\mathrm{H}_{\mathrm{b}}$ around $1.7 \AA$ are plotted as a function of applied potential. As seen in experiments, Pt-100 shows an initial response followed by Pt-111 and Pt$110 .{ }^{9}$ 
A bound hydrogen is shown on different planes Pt-111, Pt-100, and Pt-110 in figure 4C. The minimum energy sites seen here, hollow sites for both Pt-111 and Pt-100, and a lower bridge site for Pt-110 match well with the DFT calculations. ${ }^{19} \mathrm{Pt}-\mathrm{H}$ formation is the first step of the HER process, and is called the Volmer step in that context. Subsequent $\mathrm{H}_{2}$ formation can follow either of Heyrovsky or Tafel steps. ${ }^{5}$
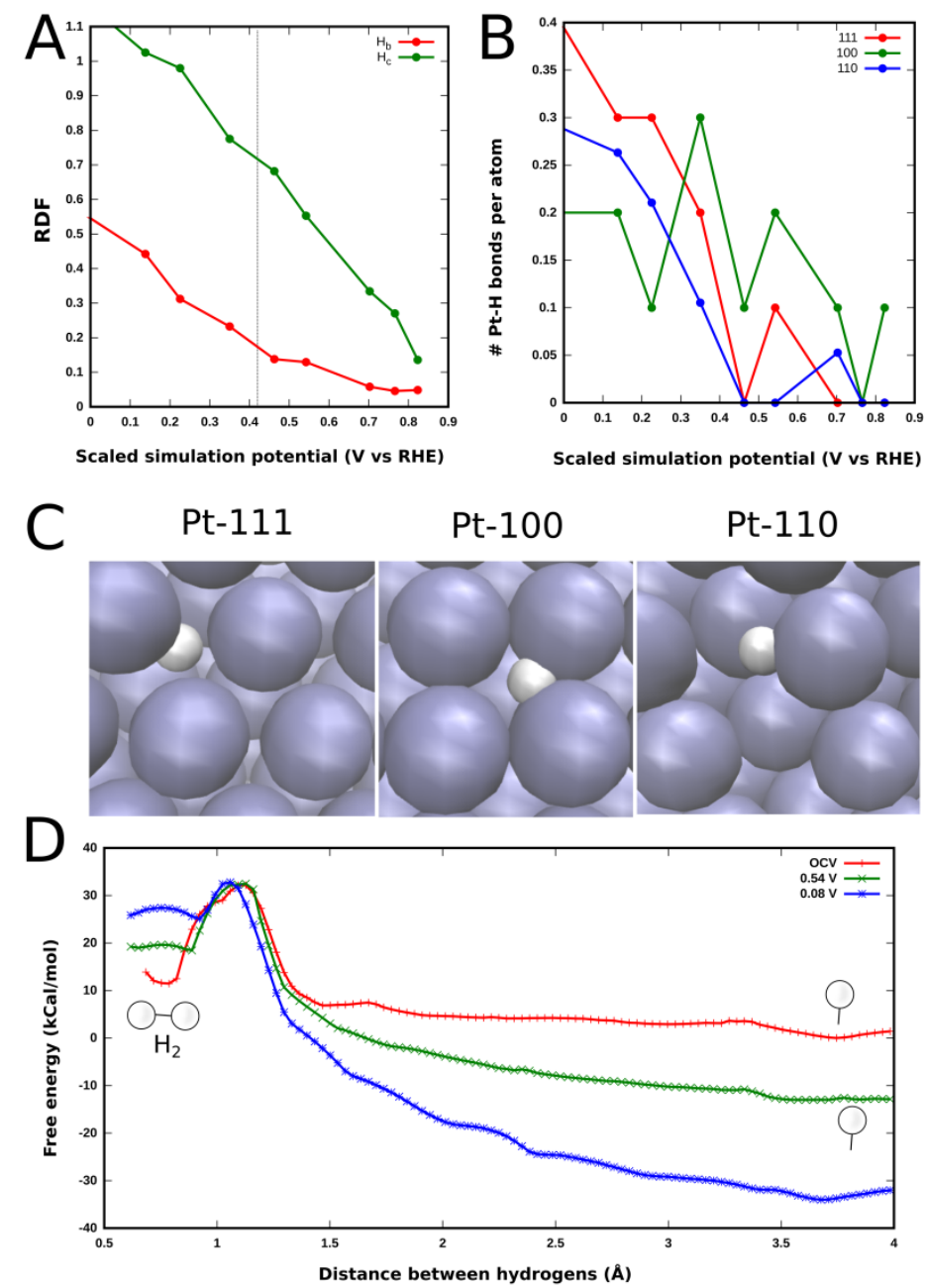

Figure 4: A) Radial distribution function values for the Pt-H interaction at $1.7\left(\mathrm{H}_{\mathrm{b}}\right)$ and $2.1\left(\mathrm{H}_{\mathrm{c}}\right) \AA$ (from figure 3D), as a function of simulation potential. B) Plane dependent hydrogen binding for 111, 100, and 110 planes with simulated potential. C) Snapshots of an absorbed hydrogen atom 
(white) on 111, 100, and 110 platinum planes. D) Free energy profile of HER/HOR, at different simulated potentials.

Although Pt-H binding (Volmer step) is observed, no subsequent HER reactions (Heyrovsky/Tafel) are seen in unbiased simulations. A low energy barrier for the Volmer step is predicted in DFT calculations, ${ }^{7}$ which is in agreement with the high binding affinity seen here. The formed Pt-H bond does not break easily, as we do not observe any desorption even if hydrogen atoms are artificially planted on the surface. The free energy barriers measured in DFT calculations for Heyrovsky/Tafel steps also show a significantly larger barrier compared to the Volmer step. ${ }^{7}$

To simulate the HER/HOR free energy profile, Umbrella sampling simulations are performed which can bias the $\mathrm{H}-\mathrm{H}$ distance and sample the free energy from decoupled Pt-H bonds to $\mathrm{H}_{2}$ formation (figure 4D). From two hydrogen atoms adsorbed on adjacent platinum bridge positions ( $\sim 4.0 \AA$ ) we sample the distance till a H-H binding of $\sim 0.75 \AA$. When the hydrogen coverage is low, hydrogen atoms prefer to be separated by a distance $\sim 4.0 \AA$. The free energy landscape for the Tafel reaction at different potentials $(\mathrm{OCV}, 0.54,0.08 \mathrm{~V})$ is shown in figure $4 \mathrm{D}$, wherein the profile is normalised to the barrier height at OCV. A barrier around $1.25 \AA$ can be seen for the HER/HOR process, during H-H bond breaking/formation. The free energy barrier for the HER is found to be $\sim 30 \mathrm{kCal} / \mathrm{mol}$ at $\mathrm{OCV}$ and increases up to $\sim 60 \mathrm{kCal} / \mathrm{mol}$ as an external potential is applied. This high barrier may be due to repulsion between two positively charged $\mathrm{H}_{b}$ on a negatively charged platinum surface. Since the HER is coverage dependent, only at high coverages of $\mathrm{H}_{\mathrm{b}}$ the repulsion may be minimised to evolve $\mathrm{H}_{2}$. And such high coverages cannot be realised in these simulations, as the hydronium ion concentration is limited, and $a<30 \%$ coverage is seen (figure 4B). Due to this, these barriers are very large compared to the enthalpy of activation calculated from experiments for the HER/HOR, $9.5-18 \mathrm{~kJ} / \mathrm{mol}(2-4 \mathrm{kCal} / \mathrm{mol}) .{ }^{9}$ DFT simulations 
using a nudged elastic band approach find barrier heights of $\sim 0.5 \mathrm{eV}(11-12 \mathrm{kCal} / \mathrm{mol})$ from an undefined initial state, which are also very high. ${ }^{7}$ The reverse process HOR, however, is not coverage dependent, and a decrease in the barrier height can be seen for a free hydrogen to dissociated state, from a barrier of $\sim 20 \mathrm{kCal} / \mathrm{mol}$ to $\sim 6 \mathrm{kCal} / \mathrm{mol}$, when an external potential close of $0.08 \mathrm{~V}$ is applied. This compares well with the activation energy in experiments, at the potential $(\sim 0 \mathrm{~V})$ where HOR is expected (figure $1 \mathrm{~A}) .^{9}$

d) Anodic reactions: Platinum Oxide Formation and Leaching:On the anodic (positive potential) side, we see prominent Pt-O bonding. Starting with a monolayer of water molecules, we see preferential Pt-O formation on the anode in the form of a capacitive layer $\sim 2.1 \AA$ and bound Pt-O $\sim 1.7 \AA$ (figure $3 \mathrm{~A}$ ). PtO surface oxide formation is seen in experiments at high anodic potentials starting close to 1.2-1.3 V (figure 1C). In our simulations, this Pt-O formation is seen close to 1.1$1.2 \mathrm{~V}$, which compare well with experiments (figure 5A). In situ Raman spectroscopy predicts the formation of alpha- $\mathrm{PtO}_{2}$ surface oxide above $1.3 \mathrm{~V}$ vs RHE. ${ }^{13}$ The platinum oxide formed in our simulations forms an amorphous layer on top, and certain platinum atoms are discernible with peroxo linkages (figure 5B).

$\mathrm{PtO}$ formation has been considered as a possible reason for leaching, where a platinum ion or an oxidised platinum cluster gets detached from the surface of the electrode (figure 5C). Positive charge accumulation disrupts the top platinum layer and a lot of rearrangement is visible (figure 2B and supporting information figure, figure $\mathrm{S} 1$ ), with preferential platinum leaching observed on the anode. Coordination of the platinum plays a big role in the charge accumulated and thus the chemical interactions. A lower coordinated platinum accumulates higher charge (figure 2D), and also interacts more with the solvent due to accessibility. The coordination of platinum atoms 
decreases in the order, $111>100>110>$ edges. Although lower coordination affords higher activity, the stability is compromised. In simulations, leaching in polycrystalline $\mathrm{Pt}(\mathrm{Pt}-\mathrm{PC})$ is observed around $\sim 1.2 \mathrm{~V}$, which compares well with 1.0-1.2 V, found by measuring dissolved platinum amounts after long time chronoamperometry at a particular potential. ${ }^{14}$ In the constant potential mode, we observe platinum leaches only at $\sim 1.2 \mathrm{~V}$, after which interstitial oxide expands and stabilise the surface (figure S1). A similar potential is applied on conical platinum electrodes with only specific planes of 111,100 , and 110 , as shown in figure S2. At $\sim 1.2 \mathrm{~V}$, we observe higher leaching off in 110 and 100 planes, compared to that in 111 (figure S3). Ultimately 2-3 atoms are seem to be leached out of the 364 atoms containing electrode, in the observed simulation time of $\sim 300$ ps. Edge atoms predominantly leach out from the surface, which can lead to a distortion of the surface and further leaching. Hence, the stability of the Pt atoms in the edges between two planes plays a crucial role to reduce the leaching off, which is shown to be highly stable for Pt-111. 

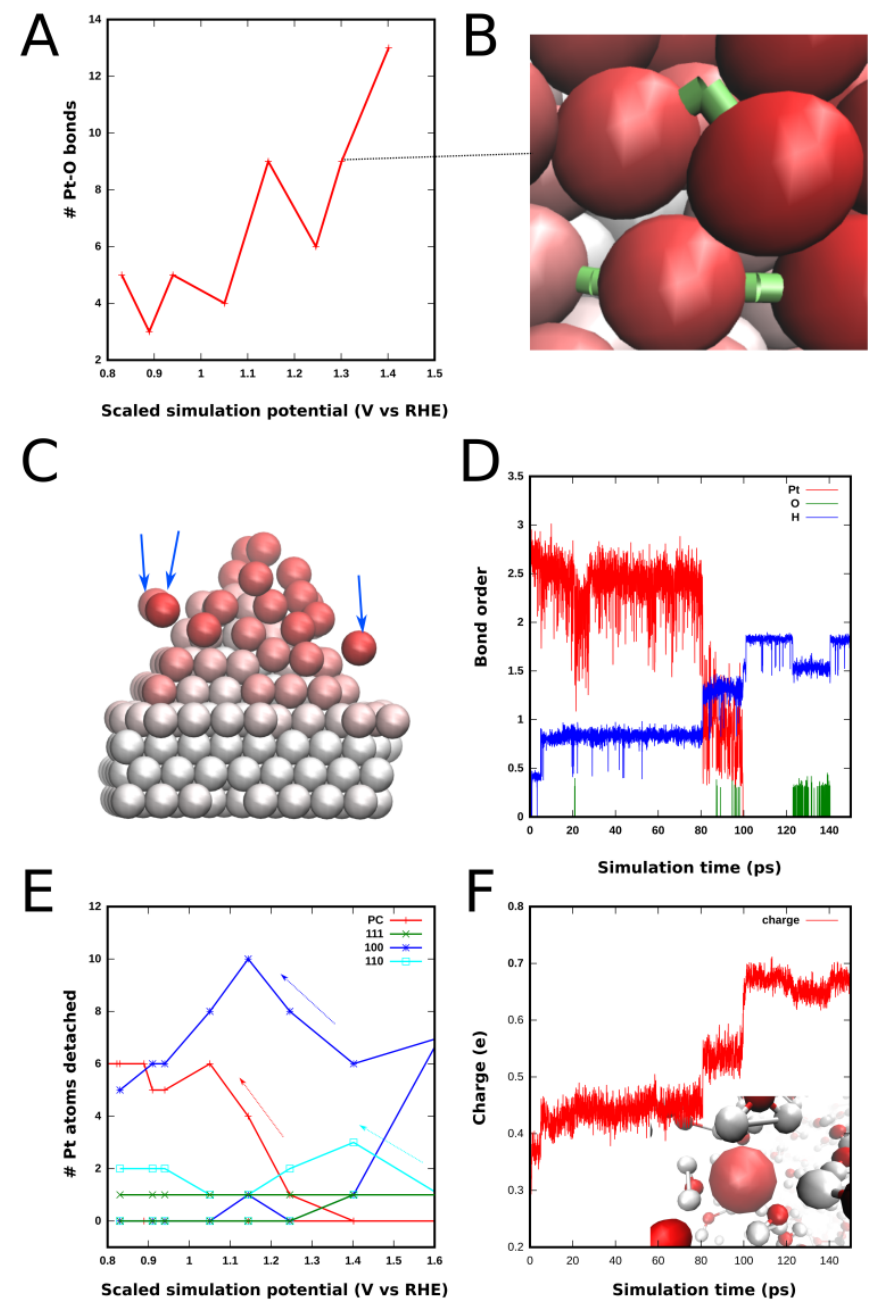

Figure 5: A) Number of Pt-O bonds formed at different simulated potentials and B) platinum oxide formation at a particular potential (oxygen bonds shown in green). C) Snapshot showing leached out (off) platinum atoms from the positively charged anode. A time profile of bond order (D) and charge (F) for a particular leached platinum atom, shown in F (inset). E) Number of platinum atoms leached off from different electrodes, polycrystalline (PC), 111, 100, and 110 surfaces, as the potential is swept from left to right and back.

Figures $5 \mathrm{D}$ and $5 \mathrm{~F}$ plot the bonding and charge profile of a leached platinum atom across simulation time. A lower coordinated platinum (bond order of 2.5-3) coordinates with hydrogen 
atoms of water in steps. Four distinct steps of coordination with four hydrogen atoms can be seen, resulting in the formation of a platinum ion coordinated with 4-8 water molecules (figure $5 \mathrm{~F}$ inset). The charge also builds up as the platinum ion binds to water and detaches from the surface. A similar behavior is seen in other leached platinum ions too (figure S4).

Apart from leaching off studies via chronoamperometry, Lopes et. al. also find cycling dependent leaching. ${ }^{15}$ In this case, when a platinum electrode is cycled to a positive potential, in the reverse scan spontaneous leaching off of platinum ions is observed through ICP-MS. ${ }^{15} \mathrm{~A}$ stepwise potential is simulated here to recreate this study, from the OCV to a high anodic potential voltage steps (of $1 \mathrm{~V}_{\text {sim }}$ ) are simulated where each step comprises of $\sim 20$ ps of simulation time. The number of Pt atoms detached from the anode as a function of cycling potential is shown in figure 5E, for the polycrystalline (PC), 111, 100, and 110 planes shown in figure S2. Pt-PC, 110, and 100 surfaces show a far more leaching off than Pt-111 in these simulations. While small amount of leaching off is observed near $\sim 1.3 \mathrm{~V}$ and at higher potentials, the reverse scan is much more destructive for the Pt surface. A higher leaching off can be ascribed to platinumds oxide formation, which destabilises the surface when swept in the reverse potential. A re-deposition of platinum ions can also be seen here, which corresponds to platinum ions adsorbed in the solvation layer and not attached on the surface. Platinum can be expected to leach off in the form of single ions at lower anodic potentials (seen here), or in the form of platinum oxide clusters at higher potentials (and higher oxide coverage). ${ }^{20}$ 


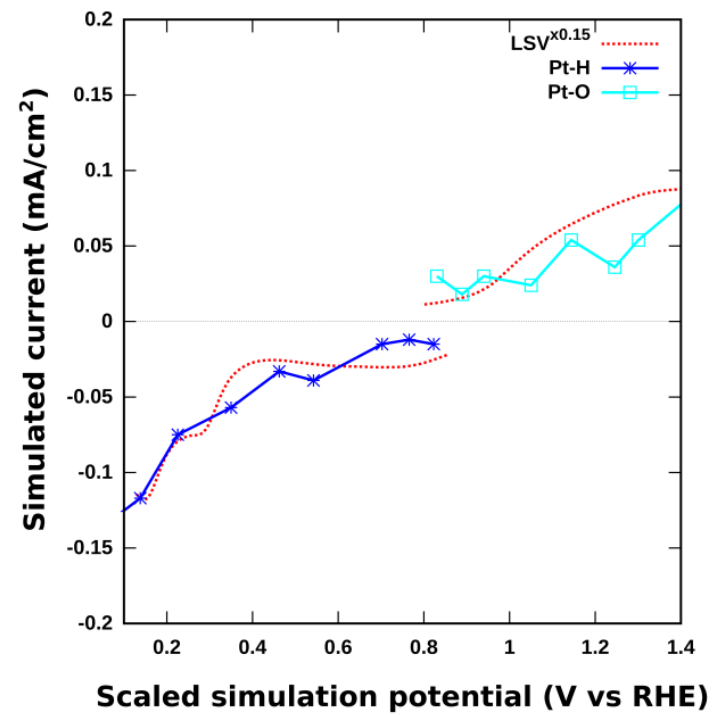

Figure 6: Simulated I-V plot (light and dark blue) showing current (derived from charge transfer) as a function of applied potential for Pt-H and Pt-O bonds, overlayed with experimental LSV plots (red) for oxidation and reduction from OCV (which is scaled $0.15 \mathrm{x}$ for comparison).

Overall, we observe potential dependent Pt-H formation (figure 4) on the cathode and Pt-O formation (figure 5) on the anode, at scaled potentials comparable to experiments. Assuming $1 \mathrm{e}^{-}$ transfer for each Pt-H formation and $2 \mathrm{e}^{-}$transfer for each Pt-O formation step, simulated LSVs are plotted in figure 6 . Along with this, experimental LSVs (red dotted line) are scaled by $0.15 \mathrm{x}$ and plotted alongside simulated I-V plots. The potential dependence seen here correlates well with the expected experimental potentials for $\mathrm{H}_{\mathrm{UPD}}$ and platinum oxide formation, which can be thought of as activation steps for HER and OER, respectively.

To conclude, various electrochemical processes on the platinum surface are studied here. By simulating an applied potential, contrasting chemical transformations on the cathode and anode are seen. On the cathode, as an external potential is applied, a negative charge distribution is adopted and preferential binding to hydrogen is seen ( $\sim 0.3$ to $0.0 \mathrm{~V}$ vs RHE), superseded by the HER/HOR $(\sim 0 \mathrm{~V})$. The anode, on the other hand, acquires a positive charge and binds to oxygen 
$(>1.2 \mathrm{~V})$, which subsequently forms $\mathrm{O}_{2}$ in the OER $(>1.6 \mathrm{~V})$. In both the cases, the potential and crystallographic orientation dependence of $\mathrm{Pt}-\mathrm{H}$ and $\mathrm{Pt}-\mathrm{O}$ intermediates are also shown, where they are in tune with the experimental reports. Apart from these processes, Pt leaching off is also studied where it preferentially occurs from the anode surface, and its crystallographic orientation dependence is also established (Pt-110 \& Pt-100 > Pt-111). Overall, a complete atomistic picture of the potential dependent long-timescale dynamics and bonding of the Pt-electrolyte interface is elucidated. These theoretical findings are in tune with the experimental potential and/or plane dependence of $\mathrm{H}_{\mathrm{UPD}}$, Pt-O, etc., indicating the potential of this platform in understanding the complex different time and length scales involved electrode-electrolyte phenomena of different systems using a computationally viable method.

\section{Experimental Methods:}

Electrochemistry: A platinum rotating disc electrode of $\sim 0.1884 \mathrm{~cm}^{2}$ area is used for the electrochemical studies presented here. Biologic-300 potentiostat is for the current and voltage controlled measurements. A three electrode system consisting Pt working and counter electrodes and an $\mathrm{Ag} / \mathrm{AgCl}$ reference electrode are used. $0.1 \mathrm{M}$ acetate buffer is used as electrolyte to keep a $\mathrm{pH} \sim 5$ for all the studies. Initial activation and subsequent $\mathrm{CVs}$ are carried out at $200 \mathrm{mV} / \mathrm{s}$, followed by pulsed voltammetry from open circuit voltage (OCV). Pulse voltammetry, in the form of a 5 second working pulse from OCV to desired potential, and 4 second reverse pulse back to OCV are applied, in $100 \mathrm{mV}$ steps (figure 1E). A total of 10 steps from OCV are recorded, towards positive (upto $\sim 2.0 \mathrm{~V}$ ) and negative regions (upto $\sim-0.1 \mathrm{~V}$ ), with the current being recorded every 
0.0002 seconds. Charge is calculated by integrating the current pulse to the final saturated current. ${ }^{16}$

MD Simulation: 792 platinum atoms forming a conical cathode and anode are kept in a cell size of $2.24 \times 1.94 \times 6.62 \mathrm{~nm}^{3}$ cell (figure 2A). 20 molecules of carbonic acid, in the form of $\mathrm{CO}_{3}{ }^{-}$and $\mathrm{H}_{3} \mathrm{O}^{+}$are added and the box is solvated to simulate a $\mathrm{pH} \sim 5$. All simulations are performed using the open source MD program LAMMPS. ${ }^{22}$ Bonding and non bonding are parameterised using the ReaxFF force field for $\mathrm{Pt} / \mathrm{C} / \mathrm{H} / \mathrm{O} \cdot{ }^{17}$ Apart from bonding parameters, which are dependent on the bond order, charge is modeled using the charge equilibration method (Qeq). In this method, the electronic energy is calculated as $\sum \chi_{i} q_{i}+\frac{1}{2} H_{i} q_{i}^{2}+\mathrm{J}_{\text {coul }}$, where the overall charge depends on the electronegativity $\left(\chi_{0}\right)$, hardness $\left(H_{0}\right)$, and the coulombic interactions $\left(\mathrm{J}_{\text {coul }}\right)$ with other atoms. Here, electronegativity $\left(\chi_{0}\right)$ and hardness $\left(H_{0}\right)$ are empirical parameters which are parameterised in ReaxFF. An external potential can applied using the EchemDID module in LAMMPS, which changes the electronegativity $\left(\chi_{0}\right)$ of particular atoms, in the QEq electronic energy. ${ }^{11}$ By setting the electronegativity $\left(\chi_{0}\right)$ of the anode to $\chi_{0}+\frac{1}{2} V$, and the cathode to $\chi_{0}-\frac{1}{2} V$, a potential of V can be applied between the two electrodes. Upto 320 ps are run at a particular applied potential, in an NVT ensemble where a temperature of $300 \mathrm{~K}$ is maintained using the Berendsen thermostat. ${ }^{23}$

\section{ASSOCIATED CONTENT}

\section{Supporting Information.}

The following files are available free of charge.

Catalyst surface structure at different simulated potentials, surface morphology of Pt-111, 100, and 110 single crystal lattices, leaching of platinum atoms for different surfaces. [SI.pdf] 


\section{AUTHOR INFORMATION}

\section{Corresponding Author}

* Corresponding Authors: sumit.bawari@gmail.com (S.B.), tnn@tifrh.res.in (T.N.N.), and jmondal@tifrh.res.in (J.M.)

\section{ACKNOWLEDGMENT}

Authors acknowledge the support of the Department of Atomic Energy, Government of India, under Project Identification No. RTI 4007.

\section{REFERENCES}

(1) She, Z. W.; Kibsgaard, J.; Dickens, C. F.; Chorkendorff, I.; Nørskov, J. K.; Jaramillo, T. F. Combining Theory and Experiment in Electrocatalysis: Insights into Materials Design. Science2017, 355 (6321), eaad4998.

(2) Nitopi, S.; Bertheussen, E.; Scott, S. B.; Liu, X.; Engstfeld, A. K.; Horch, S.; Seger, B.; Stephens, I. E. L.; Chan, K.; Hahn, C.; et al. Progress and Perspectives of Electrochemical $\mathrm{CO}_{2}$ Reduction on Copper in Aqueous Electrolyte. Chem. Rev.2019, 119 (12), 7610-7672.

(3) Shi, L.; Yin, Y.; Wang, S.; Sun, H. Rational Catalyst Design for $\mathrm{N}_{2}$ Reduction under Ambient Conditions: Strategies toward Enhanced Conversion Efficiency. ACS Catal.2020, 10 (12), 68706899.

(4) Scalfi, L.; Salanne, M.; Rotenberg, B. Molecular Simulation of Electrode-Solution Interfaces. Annu. Rev. Phys. Chem.2021, 72 (1), 090519-024042. 
(5) Bawari, S.; Narayanan, T. N.; Mondal, J. Atomistic Elucidation of Sorption Processes in Hydrogen Evolution Reaction on a van Der Waals Heterostructure. J. Phys. Chem. C2018, 122 (18), 10034-10041.

(6) Rebollar, L.; Intikhab, S.; Oliveira, N. J.; Yan, Y.; Xu, B.; McCrum, I. T.; Snyder, J. D.; Tang, M. H. “Beyond Adsorption” Descriptors in Hydrogen Electrocatalysis. ACS Catal.2020, 10 (24), 14747-14762.

(7) Skúlason, E.; Karlberg, G. S.; Rossmeisl, J.; Bligaard, T.; Greeley, J.; Jónsson, H.; Nørskov, J. K.; Skulason, E.; Karlberg, G. S.; Rossmeisl, J.; et al. Density Functional Theory Calculations for the Hydrogen Evolution Reaction in an Electrochemical Double Layer on the Pt(111) Electrode. Phys. Chem. Chem. Phys.2007, 9 (25), 3241-3250.

(8) Merlet, C.; Rotenberg, B.; Madden, P. A.; Taberna, P. L.; Simon, P.; Gogotsi, Y.; Salanne, M. On the Molecular Origin of Supercapacitance in Nanoporous Carbon Electrodes. Nat. Mater.2012, 11 (4), 306-310.

(9) Marković, N. M.; Grgur, B. N.; Ross, P. N. Temperature-Dependent Hydrogen Electrochemistry on Platinum Low-Index Single-Crystal Surfaces in Acid Solutions. J. Phys. Chem. B1997, 101 (27), 5405-5413.

(10) Cheng, T.; Wang, L.; Merinov, B. V.; Goddard, W. A. Explanation of Dramatic PHDependence of Hydrogen Binding on Noble Metal Electrode: Greatly Weakened Water Adsorption at High PH. J. Am. Chem. Soc.2018, 140 (25), 7787-7790.

(11) Onofrio, N.; Strachan, A. Voltage Equilibration for Reactive Atomistic Simulations of Electrochemical Processes. J. Chem. Phys.2015, 143 (5). 
(12) Onofrio, N.; Guzman, D.; Strachan, A. Atomic Origin of Ultrafast Resistance Switching in Nanoscale Electrometallization Cells. Nat. Mater.2015, 14 (4), 440-446.

(13) Huang, Y. F.; Kooyman, P. J.; Koper, M. T. M. Intermediate Stages of Electrochemical Oxidation of Single-Crystalline Platinum Revealed by in Situ Raman Spectroscopy. Nat. Commun.2016, 7, 1-7.

(14) Sugawara, Y.; Okayasu, T.; Yadav, A. P.; Nishikata, A.; Tsuru, T. Dissolution Mechanism of Platinum in Sulfuric Acid Solution. J. Electrochem. Soc.2012, 159 (11), F779-F786.

(15) Lopes, P. P.; Strmcnik, D.; Tripkovic, D.; Connell, J. G.; Stamenkovic, V.; Markovic, N. M. Relationships between Atomic Level Surface Structure and Stability/Activity of Platinum Surface Atoms in Aqueous Environments. ACS Catal.2016, 6 (4), 2536-2544.

(16) Nong, H. N.; Falling, L. J.; Bergmann, A.; Klingenhof, M.; Tran, H. P.; Spöri, C.; Mom, R.; Timoshenko, J.; Zichittella, G.; Knop-Gericke, A.; et al. Key Role of Chemistry versus Bias in Electrocatalytic Oxygen Evolution. Nature2020, 587 (7834), 408-413.

(17) Shin, Y. K.; Gai, L.; Raman, S.; Van Duin, A. C. T. Development of a ReaxFF Reactive Force Field for the Pt-Ni Alloy Catalyst. J. Phys. Chem. A2016, 120 (41), 8044-8055.

(18) Ogasawara, H.; Brena, B.;Nordlund, D.; Nyberg, M.; Pelmenschikov, A.; Pettersson, L. G. M.; Nilsson, A. Structure and Bonding of Water on Pt(111). Phys. Rev. Lett.2002, 89 (111), 276102.

(19) Vurdu, C. D. The Adsorption and Diffusion Manners of Hydrogen Atoms on Pt (100), Pt (110), and Pt (111) Surfaces. Adv. Condens. Matter Phys.2018, 2018 (110). 
(20) Kirchhoff, B.; Braunwarth, L.; Jung, C.; Jónsson, H.; Fantauzzi, D.; Jacob, T. Simulations of the Oxidation and Degradation of Platinum Electrocatalysts. Small2020, 16 (5).

(21) Guha, A.; Veettil Vineesh, T.; Sekar, A.; Narayanaru, S.; Sahoo, M.; Nayak, S.; Chakraborty, S.; Narayanan, T. N. Mechanistic Insight into Enhanced Hydrogen Evolution Reaction Activity of Ultrathin Hexagonal Boron Nitride-Modified Pt Electrodes. ACS Catal.2018, $8(7), 6636-6644$.

(22) Aktulga, H. M.; Fogarty, J. C.; Pandit, S. A.; Grama, A. Y. Parallel Reactive Molecular Dynamics: Numerical Methods and Algorithmic Techniques. Parallel Comput.2012, 38 (4-5), $245-259$.

(23) Berendsen, H. J. C.; Postma, J. P. M.; van Gunsteren, W. F.; DiNola, A.; Haak, J. R. Molecular Dynamics with Coupling to an External Bath. J. Chem. Phys.1984, 81 (8), 3684-3690. 


\section{Supporting Information}
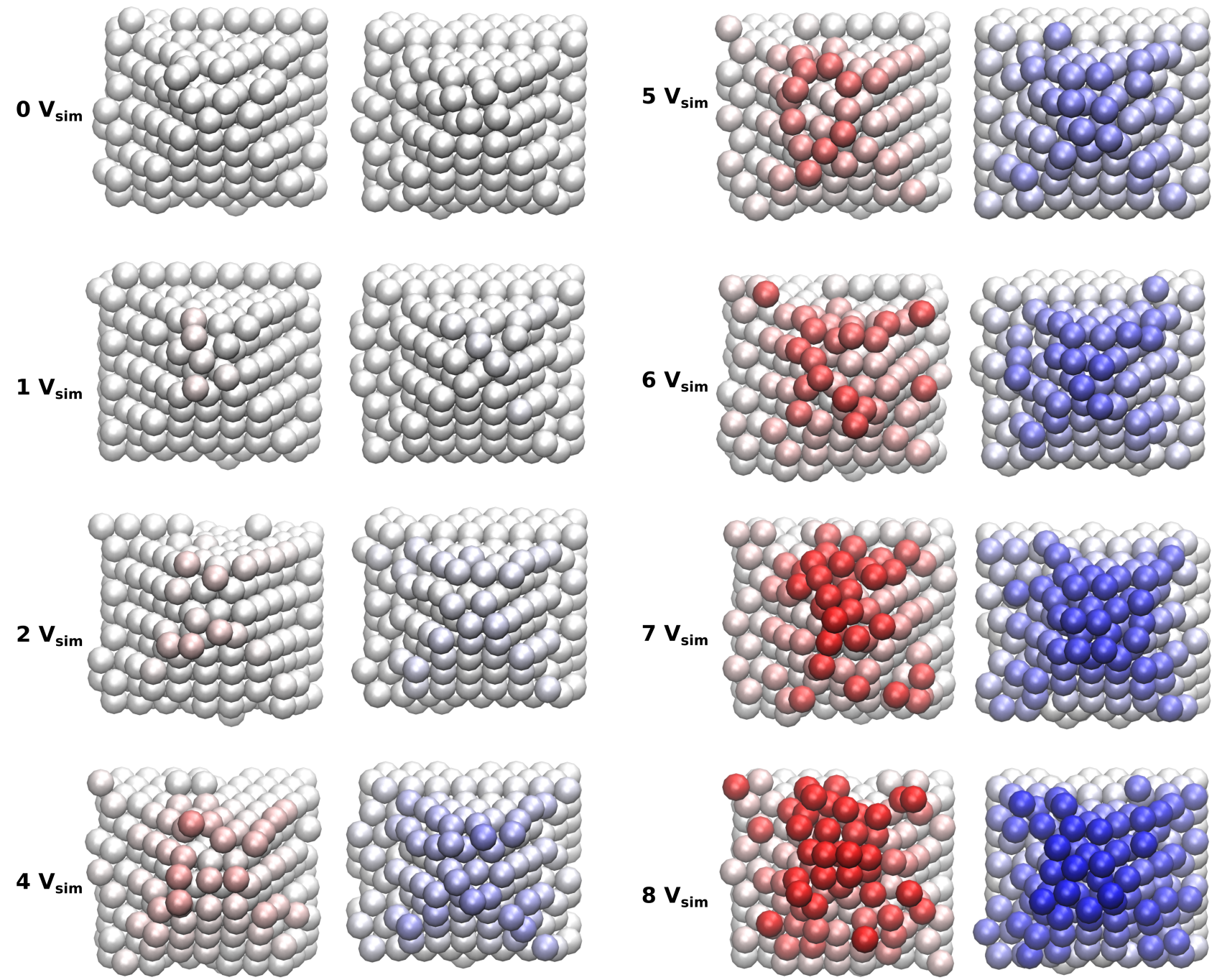

Figure S1: Snapshots of simulated electrodes at different potentials (in $\mathrm{V}_{\text {sim }}$ ) 
Pt-111

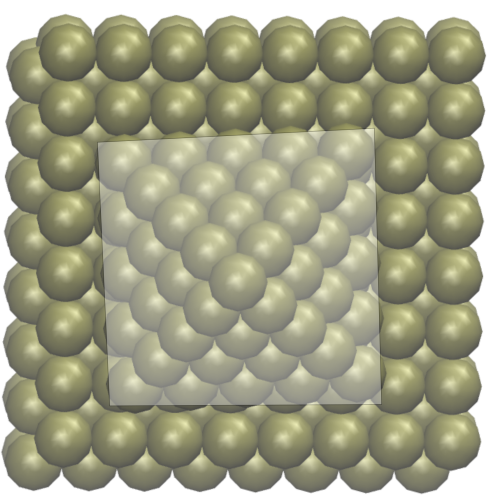

Pt-100

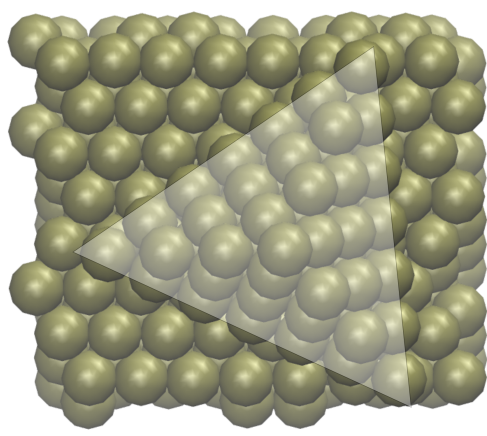

Pt-110

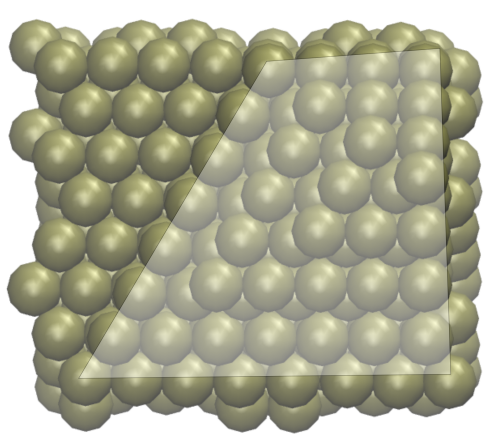

Figure S2: Morphology of specific platinum planes, 111, 100 and 110, used in simulations, with the protruding section highlighted. 


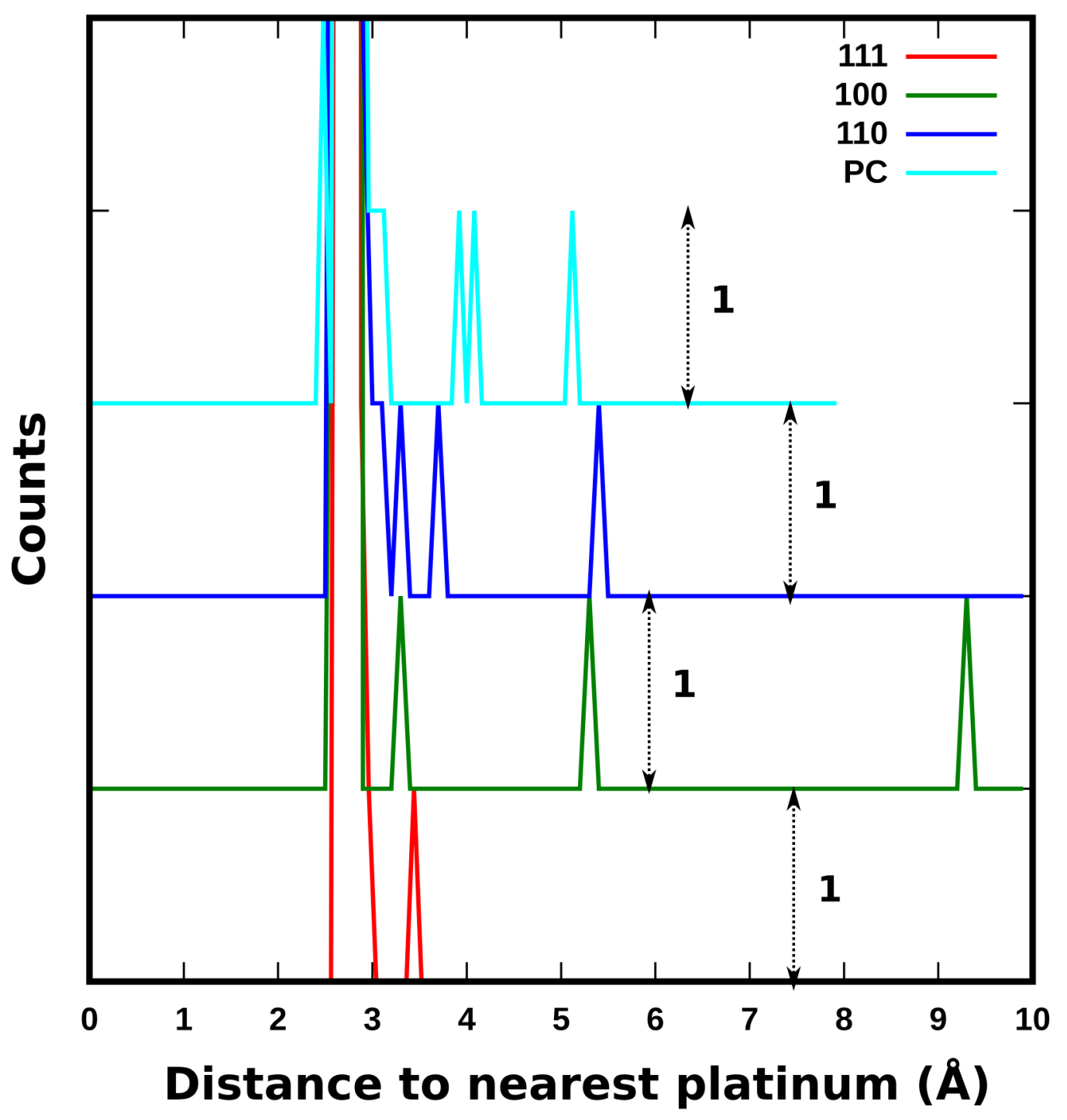

Figure S3: Distance of all platinum atoms in the anode to its nearest neighbour after a $320 \mathrm{ps}$ simulation at $6 \mathrm{~V}_{\text {sim }}$ potential. Any peaks more than $\sim 3$ Angstrom signify a detached platinum atom. 